\title{
Experimental infection with Bordefella bronchiseptica and Pasteurella multocida for a better control of vaccines against atrophic rhinitis in the pig
}

\author{
Marylène KOBISCH \\ Ministère de l'Agriculture, Station de Pathologie Porcine \\ B.P. 9, Les Croix, 22440 Ploufragan
}

Thirty-seven piglets born from 4 SPF (Specific Pathogen Free) sows were infected intranasally with Bordetella bronchiseptica at two days of age and 5 days later with a toxigenic Pasteurella multocida type D. Thirteen SPF piglets born from 2 other sows were used as control group. One third of piglets from each group were sacrificed 3 to 10 weeks after infection.

Eight piglets died from 1 to 17 days after challenge. Clinical symptoms (particularly sneezing) occurred after some days and persisted during the whole experimental period. Macroscopic examinations indicated severe pneumonia in 68 p. 100 of the infected piglets. Changes in the turbinates were visible 3 days after challenge, then deviation of the snout and of the nasal septum as well as complete turbinate atrophy were observed. The value of brachignathia was $2.5 \mathrm{~cm}$ at the end of the experiment. Antibodies were detectable in sows and in piglets.

This experimental model could be useful to evaluate the efficacy of vaccines against atrophic rhinitis.

\section{Comparison between functional properties of alveolar macrophages and immunoglobulin concentrations of tracheobronchial secretions in Large White and Mei-Shan pigs}

\author{
J.M. SACHOT *, S. BERNARD *, Elizabeth BOTTREAU *, \\ Christiane de VAUREIX **, B. CHARLEY ** \\ * I.N.R.A., Laboratoire de Pathologie Porcine, 37380 Nouzilly \\ ** Station de Virologie et d'Immunologie, 78850 Thivernal-Grignon
}

It was observed that Chinese pig breeds introduced in France are more susceptible to respiratory diesases than European breeds. This sensitivity was attributed to a lower activity of alveolar macrophages (AM). To confirm this hypothesis a study was made to compare the functionnal properties in vitro of AM in Large White (LW) and Mei-Shan (MS) piglets kept in the same place. Immunoglobulin (Ig) concentrations were also compared as they play a role in the defense of the respiratory system. AM were obtained by lung lavage post mortem. Ig concentrations were determined using the ELISA technique.

Our results showed significant differences in favour of the Mei-Shan breed for the following properties : spreading, adherence, phagocytosis of sensitized sheep red blood cells (SRBC) and resistance to transmissible gastro-enteritis virus. On the other hand, there was no difference between the two breeds as regards $\mathrm{Ig}$ concentrations in tracheobronchial secretions, viability of $A M$ and phagocytic and bactericidal capacity of AM towards Listeria monocytogenes.

It was concluded that the higher susceptibility of Chinese pigs to respiratory diseases is not due to defective functional properties of AM. Some of these properties are even higher in MS than in LW pigs. Further studies should be made to confirm our results. 